\title{
Delta operation on modules, prime and radical submodules and primary decomposition
}

\author{
Ashkan Nikseresht (D) \\ Department of Mathematics, College of Sciences, Shiraz University, 11457-13565, Shiraz, Iran
}

\begin{abstract}
Let $R$ be a commutative ring with identity and $M$ be an $R$-module. In this paper, in order to study prime submodules, radical submodules and primary decompositions in finitely generated free $R$-modules, we introduce and study an operation $\Delta:(M \oplus R)^{2} \rightarrow M$ defined by $\Delta\left(m+r, m^{\prime}+r^{\prime}\right)=r^{\prime} m-r m^{\prime}$. In particular, using this operation we give a characterization of prime submodules of $M \oplus R$, in terms of prime submodules of $M$. As an application, we present a characterization of prime submodules of finitely generated free modules. Also we present a formula for the prime radical of submodules of $M \oplus R$. Moreover, we state some conditions under which primary decompositions of submodules of $M$ lift to $M \oplus R$.
\end{abstract}

Mathematics Subject Classification (2010). 13C99, 13A15, 13C13

Keywords. Delta operation, primary decomposition, prime submodule, radical of submodules

\section{Introduction}

In this paper all rings are commutative with identity, all modules are unitary, $R$ denotes a ring and $M$ denotes an $R$-module. Also by $\mathbb{N}$ we mean the set of positive integers. We indicate the relation of containment and strict containment by $\subseteq$ and $\subset$, respectively. Furthermore $N \leq M$ (resp., $N<M$ ) means that $N$ is a submodule (resp., proper submodule) of $M$.

Prime ideals of rings play an important role in commutative ring theory; hence many have tried to generalize this concept to modules. A proper submodule $P$ of $M$ is called prime, when from $r m \in P$ for some $r \in R$ and $m \in M$, we can conclude either $m \in P$ or $r M \subseteq P$ (see for example [1,3,4,8,14,22]). Let $(P: M)$ be the set of all $r \in R$ such that $r M \subseteq P$. If $P$ is a prime submodule, then $\mathfrak{p}=(P: M)$ is a prime ideal of $R$ and we say that $P$ is $\mathfrak{p}$-prime.

If $N$ is a submodule of $M$, the intersection of prime submodules of $M$ containing $N$ is called the radical of $N$ and we denote it by $\operatorname{rad}_{M}(N)(\operatorname{or} \operatorname{rad}(N)$ if there is no subtlety). If there is no prime submodule containing $N$, we set $\operatorname{rad}(N)=M$. Many researchers have studied and tried to give formulations for the prime radical of submodules, see for example $[2,3,5,6,9-13,16-21]$.

Email address: ashkan_nikseresht@yahoo.com

Received: 29.03.2018; Accepted: 10.09.2019 
As prime and primary submodules and radical of submodules behave well under taking quotients, it is important to characterize such submodules in free modules. In [8] a characterization of prime submodules in the $R$-module $F=R \oplus R$ is given. Also some criteria on submodules of $F$ is stated for having a primary decomposition.

The main tool in proving these results is a function $\Delta: F^{2} \rightarrow R$ defined as $\Delta\left(\left(x_{1}, x_{2}\right),\left(y_{1}, y_{2}\right)\right)=x_{1} y_{2}-y_{1} x_{2}$. In particular, they prove that for a submodule $N$ of $F$ containing neither $(1,0)$ nor $(0,1)$, being prime is equivalent to $(N: F)=\mathfrak{p}$ being a prime ideal and $N=\mathfrak{p} \oplus \mathfrak{p}$ or $N=\Delta^{\mathfrak{p}}(a, b)$ with $R a+R b \nsubseteq \mathfrak{p}$, where $\Delta^{\mathfrak{p}}(a, b)=\{(x, y) \in F \mid \Delta((x, y),(a, b)) \in \mathfrak{p}\}$. In [15], by replacing this $\Delta$ function with minors of certain matrices, the results of [8] are generalized to finitely generated free modules.

The $\Delta$ function mentioned above had been previously proved to be useful in studying prime and radical submodules. For example the $\Lambda$ operation defined by Man in $[11,12]$ and used to characterize domains satisfying specific formulas on radical of submodules, is indeed $\Lambda(a, b)=\Delta^{0}(a, b)$.

The main aim of this research is to generalize these results to every finitely generated free module. To this end we first investigate the following generalization of the $\Delta$ operation of $[8]$.

Definition 1.1. For an $R$-module $M$ let $\widetilde{M}=M \oplus R$ and define $\Delta_{M, R}: \widetilde{M}^{2} \rightarrow M$ by $\Delta_{M, R}\left(m+r, m^{\prime}+r^{\prime}\right)=r^{\prime} m-r m^{\prime}$ where $m+r$ denotes the element of $\widetilde{M}$ with $m \in M$ and $r \in R$. Also for $N \leq M$ and $\widetilde{m} \in \widetilde{M}$, we set $\Delta_{M, R}^{N}(\widetilde{m})=\left(\Delta_{M, R}(\widetilde{m}, \cdot)\right)^{-1}(N)$, the preimage of $N$ under the map $\Delta_{M, R}(\widetilde{m}, \cdot): \widetilde{M} \rightarrow M$. Moreover, for any $\widetilde{A} \subseteq \widetilde{M}$ and $N \leq M$ by $\Delta_{M, R}^{N}(\widetilde{A})$ we mean $\bigcap_{\widetilde{a} \in \widetilde{A}} \Delta_{M, R}^{N}(\widetilde{a})$. When there is no confusion we drop the subscripts $M, R$ and write $\Delta$ or $\Delta^{N}$.

Here first in Section 2, we state some basic properties of this $\Delta$ operation. Then in Section 3 , using the $\Delta$ operation we present a characterization of prime submodules and radical of submodules of $\widetilde{M}$ in terms of prime and radical submodules of $M$. We use this to state a characterization of prime submodules of finitely generated free modules. Finally in Section 4, we study when a primary decomposition of an $A \leq M$ 'lifts' to one for $\Delta^{A}(\widetilde{N})$ where $\widetilde{N} \leq \widetilde{M}$. We end this introduction with the following notations.

Notation 1.2. Throughout the paper, $\widetilde{M}=M \oplus R$ and its elements are written as $m+r$ with $m \in M$ and $r \in R$. Also we consider $M$ and $R$ as submodules of $\widetilde{M}$ in the natural way and denote the canonical projections $\widetilde{M} \rightarrow M$ and $\widetilde{M} \rightarrow R$ by $\pi_{1}$ and $\pi_{2}$, respectively.

\section{Basic properties of the delta operation}

We start with the following properties of the delta operation, whose easy proofs are left to the reader. Here $\left(N:_{M} I\right)=\{m \in M \mid I m \subseteq N\}$, for $N \leq M, I \leq R$. Also by $\Delta(\widetilde{A}, \widetilde{B})$ we mean the submodule generated by $\{\Delta(\widetilde{a}, \widetilde{b}) \mid \widetilde{a} \in \widetilde{A}, \widetilde{b} \in \widetilde{B}\}$, for $\widetilde{A}, \widetilde{B} \subseteq \widetilde{M}$.

Lemma 2.1. Suppose that $N, K, N_{\lambda} \leq M$ for each $\lambda \in \Lambda, I \leq R$ and $\widetilde{A} \subseteq \widetilde{B} \subseteq \widetilde{M}$. Then the following hold.

(a) $\Delta$ is an R-bilinear map.

(b) $\Delta^{N}(\widetilde{A})=\Delta^{N}(\langle\widetilde{A}\rangle) \leq \widetilde{M}$.

(c) $\Delta^{N}(\widetilde{B}) \subseteq \Delta^{N}(\widetilde{A})$.

(d) $\Delta^{N}(K \oplus I)=\left(N:_{M} I\right) \oplus\left(N:_{R} K\right)$.

(e) $\Delta^{N}(\widetilde{A})$ is the largest subset of $\widetilde{M}$ such that $\Delta\left(\widetilde{A}, \Delta^{N}(\widetilde{A})\right) \subseteq N$.

(f) $\Delta \bigcap_{\lambda \in \Lambda} N_{\lambda}(\widetilde{A})=\bigcap_{\lambda \in \Lambda} \Delta^{N_{\lambda}}(\widetilde{A})$. 
Corollary 2.2. For any $\widetilde{A} \subseteq \widetilde{M}$ and $N \leq M$ we have $\widetilde{A} \subseteq \Delta^{N}\left(\Delta^{N}(\widetilde{A})\right)$ and equality holds if and only if $\widetilde{A}=\Delta^{N}(\widetilde{B})$ for some $\widetilde{\widetilde{B}} \subseteq \widetilde{M}$. If we set $\bar{S}=\left\{\Delta^{N}(\widetilde{B}) \mid \widetilde{B} \subseteq \widetilde{M}\right\}$, then $S$ is a lattice with respect to inclusion and $\Delta^{\bar{N}}: S \rightarrow S$ is an order anti-automorphism.

Proof. The inclusion $\widetilde{A} \subseteq \Delta^{N}\left(\Delta^{N}(\widetilde{A})\right)$ and also the fact that the equality holds only if $A=\Delta^{N}(\widetilde{B})$ are clear. Conversely if $\widetilde{A}=\Delta^{N}(\widetilde{B})$, then $\widetilde{B} \subseteq \Delta^{N}\left(\Delta^{N}(\widetilde{B})\right)$ and by 2.1c we deduce that $\widetilde{A}=\Delta^{N}(\widetilde{B}) \supseteq \Delta^{N}\left(\Delta^{N}\left(\Delta^{N}(\widetilde{B})\right)\right)=\Delta^{N}\left(\Delta^{N}(\widetilde{\widetilde{A}})\right)$. Since the reverse inclusion always holds, we conclude that indeed equality holds.

Now it is clear that the map $\Delta^{N}: S \rightarrow S$ is an order reversing bijection whose inverse is again $\Delta^{N}$. Suppose that $\widetilde{B}_{1}, \widetilde{B}_{2} \subseteq \widetilde{M}$. It can readily be checked that $\Delta^{N}\left(\widetilde{B}_{1} \cup \widetilde{B}_{2}\right)=$ $\Delta^{N}\left(\widetilde{B}_{1}\right) \cap \Delta^{N}\left(\widetilde{B}_{2}\right)$ and $\Delta^{N}\left(\Delta^{N}\left(\Delta^{N}\left(\widetilde{B}_{1}\right)+\Delta^{N}\left(\widetilde{B}_{2}\right)\right)\right)$ are respectively the greatest lower bound and the least upper bound of $\Delta^{N}\left(\widetilde{B}_{1}\right)$ and $\Delta^{N}\left(\widetilde{B}_{2}\right)$. Thus $S$ is a lattice.

Next we find the ideal $\left(\Delta^{N}(\widetilde{A}): \widetilde{M}\right)$. Note that by $2.1 \mathrm{~b}$, we can assume that $\widetilde{A} \leq \widetilde{M}$.

Proposition 2.3. Suppose that $\widetilde{A} \leq \widetilde{M}, N \leq M$ and $K=\Delta(\widetilde{A}, \widetilde{A})$. Then

(a) $\left(\Delta^{N}(\widetilde{A}): \widetilde{M}\right)=\left(N:\left(\pi_{1}(\widetilde{A})+\pi_{2}(\widetilde{A}) M\right)\right) \supseteq(N: M)$;

(b) $K \subseteq \widetilde{A} \cap M$ and $(\widetilde{A}: \widetilde{M}) \subseteq \sqrt{(K: M)}$.

Proof. (a) Let $i \in I=\left(\Delta^{N}(\widetilde{A}): \widetilde{M}\right)$ and $\widetilde{a} \in \widetilde{A}$ with $\pi_{j}(\widetilde{a})=a_{j}$ for $j=1,2$. For each $m \in M$ we have $-i a_{2} m=0 a_{1}-i a_{2} m=\Delta(\widetilde{a}, i m) \in \Delta\left(\widetilde{A}, \Delta^{N}(\widetilde{A})\right) \subseteq N$. So $I \pi_{2}(\widetilde{A}) M \subseteq N$, that is, $I \subseteq\left(N: \pi_{2}(\widetilde{A}) M\right)$. Similarly $i a_{1}=\Delta(\widetilde{a}, i(0+1)) \subseteq N$ and hence $I \subseteq\left(N: \pi_{1}(\widetilde{A})\right)$.

Conversely, if $i \in\left(N:\left(\pi_{1}(\widetilde{A})+\pi_{2}(\widetilde{A}) M\right)\right), \widetilde{m} \in \widetilde{M}$ and $\widetilde{a} \in \widetilde{A}$, then

$$
\Delta(\widetilde{a}, i \widetilde{m})=i\left(\pi_{2}(\widetilde{m}) \pi_{1}(\widetilde{a})-\pi_{2}(\widetilde{a}) \pi_{1}(\widetilde{m})\right) \in i \pi_{1}(\widetilde{A})+i \pi_{2}(\widetilde{A}) M \subseteq N .
$$

Thus by definition of $\Delta^{N}$, we deduce that $i \widetilde{m} \in \Delta^{N}(\widetilde{A})$, which means $i \in I$, as required.

(b) Obviously $K \subseteq M$. Let $\widetilde{a}, \widetilde{a}^{\prime} \in \widetilde{A}$ with $\pi_{j}(\widetilde{a})=a_{j}, \pi_{j}\left(\widetilde{a}^{\prime}\right)=a_{j}^{\prime}$. Then

$$
\Delta\left(\widetilde{a}, \widetilde{a}^{\prime}\right)=a_{2}^{\prime} a_{1}-a_{2} a_{1}^{\prime}=a_{2}^{\prime}\left(a_{1}+a_{2}\right)-a_{2}\left(a_{1}^{\prime}+a_{2}^{\prime}\right)=a_{2}^{\prime} \widetilde{a}-a_{2} \widetilde{a}^{\prime} \in \widetilde{A} .
$$

Therefore, $K \subseteq \widetilde{A}$. Now suppose that $r \in(\widetilde{A}: \widetilde{M})$. Then for each $m \in M$ we have $r m \in \widetilde{A}$ and also $r=r(0+1) \in \widetilde{A}$, whence $r^{2} m=\Delta(r m, r) \in K$ and the result follows.

Later we will need the following lemmas which show how $\Delta$ behaves under localization and taking quotients.

Lemma 2.4. Suppose that $K \leq N \leq M, \widetilde{A} \leq \widetilde{M}, I \subseteq(K: M)$ and $\widehat{A}$ denotes the image of $\widetilde{A}$ under the canonical projection from $\widetilde{M} \rightarrow \widehat{M}=\frac{M}{K} \oplus \frac{R}{I}=\frac{\widetilde{M}}{K \oplus I}$. Then

$$
\Delta_{\frac{M}{K}, \frac{R}{I}}^{\frac{N}{K}}(\widehat{A})=\frac{\Delta_{M, R}^{N}(\widetilde{A})}{K \oplus I} .
$$

In particular, $N \oplus(N: M) \subseteq \Delta^{N}(\widetilde{A})=\Delta^{N}(\widetilde{A}+(N \oplus(N: M)))$.

Proof. Suppose that " ? " denotes the image of submodules of $M$ or $R$ in $\frac{M}{K}$ or $\frac{R}{I}$. As $\widetilde{A} \subseteq M \oplus R$ it follows 2.1c and d, that $K \oplus I \subseteq N \oplus(N: M) \subseteq \Delta_{M, R}^{N}(\widetilde{A})$. Let $\widetilde{m} \in \widetilde{M}$ and $\widehat{m}=\widetilde{m}+(K \oplus I)$. Then $\widehat{m} \in \Delta \frac{\bar{N}}{M}, \bar{R}(\widehat{A}) \Leftrightarrow \Delta_{\bar{M}}, \bar{R}(\widehat{a}, \widehat{m}) \in \bar{N}$ for each $\widehat{a} \in \widehat{A}$. But if $\widetilde{a} \in \widetilde{A}$ is a preimage of $\widehat{a}$, then $\Delta_{\bar{M}, \bar{R}}(\widehat{a}, \widehat{m})=\Delta_{M, R}(\widetilde{a}, \widetilde{m})+K$. Hence $\widehat{m} \in \Delta_{\bar{M}} \overline{\bar{N}}, \bar{R}(\widehat{A}) \Leftrightarrow \Delta_{M, R}(\widetilde{a}, \widetilde{m}) \in N$ for each $\widetilde{a} \in \widetilde{A} \Leftrightarrow \widetilde{m} \in \Delta_{M, R}^{N}(\widetilde{A})$. The "in particular" statement follows by setting $K=N$ and $I=(N: M)$ in the main statement.

Lemma 2.5. Assume that $S$ is a multiplicatively closed subset of $R, \widetilde{A} \leq \widetilde{M}$ and $K$ is an $S^{-1} R$-submodule of $S^{-1} M$. Then $\left(\Delta_{S^{-1} M, S^{-1} R}^{K}\left(S^{-1} \widetilde{A}\right)\right)^{c}=\Delta_{M, R}^{K^{c}}(\widetilde{A})$, where ".c " denotes contraction under the localization map. In particular, $\Delta_{M, R}^{K^{c}}(\widetilde{A})=\Delta_{M, R}^{K^{c}}\left(\left(S^{-1} \widetilde{A}\right)^{c}\right)$. 
Proof. We have

$$
\widetilde{m} \in\left(\Delta_{S^{-1} M, S^{-1} R}^{K}\left(S^{-1} \widetilde{A}\right)\right)^{c} \Leftrightarrow \Delta_{S^{-1} M, S^{-1} R}\left(\frac{\widetilde{a}}{s}, \frac{\widetilde{m}}{1}\right)=\frac{\Delta_{M, R}(\widetilde{a}, \widetilde{m})}{s} \in K,
$$

for each $\widetilde{a} \in \widetilde{A}$ and $s \in S$. This is equivalent to $\frac{\Delta_{M, R}(\widetilde{a}, \widetilde{m})}{1} \in K$, that is, $\Delta_{M, R}(\widetilde{a}, \widetilde{m}) \in K^{c}$ for all $\widetilde{a} \in \widetilde{A}$ or equivalently $\widetilde{m} \in \Delta_{M, R}^{K^{c}}(\widetilde{A})$.

A proper submodule $P$ of $M$ is called weakly prime, when from $r_{1} r_{2} m \in P$ we can deduce that either either $r_{1} m \in \mathrm{P}$ or $r_{2} m \in P$. It is easy to see that $\mathfrak{p}=(P: M)$ is a prime ideal, when $P$ is weakly prime. In this case, we say that $P$ is weakly $\mathfrak{p}$-prime. This concept was first introduced in [7] as another generalization of prime ideals. It should be mentioned that in some papers weakly prime submodules are called classical prime. The following shows that prime, weakly prime and primary submodules behave well under $\Delta$.

Theorem 2.6. Suppose that $P$ is a $\mathfrak{p}$-primary (resp. p-prime, weakly $\mathfrak{p}$-prime) submodule of $M$ and $\widetilde{N} \leq \widetilde{M}$. If $\widetilde{N} \nsubseteq P \oplus(P: M)$, then $\widetilde{D}=\Delta^{P}(\widetilde{N})$ is a $\mathfrak{p}$-primary (resp. $\mathfrak{p}$-prime, weakly prime) submodule of $\widetilde{M}$.

Proof. First note that by $2.1 \mathrm{~d}$ and also $2.2, \widetilde{D} \neq \widetilde{M}$. Suppose that $P$ is p-primary and $r \widetilde{m} \in \widetilde{D}$, where $r \in R \backslash \mathfrak{p}$ and $\widetilde{m} \in \widetilde{M}$. For each $\widetilde{n} \in \widetilde{N}$, we have $r \Delta(\widetilde{n}, \widetilde{m})=\Delta(\widetilde{n}, r \widetilde{m}) \in$ $\Delta(\tilde{N}, \widetilde{D}) \subseteq P$, by definition of $\widetilde{D}$. So by $P$ being $\mathfrak{p}$-primary and $r \notin \mathfrak{p}$, we deduce that $\Delta(\widetilde{n}, \widetilde{m}) \in P$ for each $\widetilde{n} \in \widetilde{N}$, that is, $\widetilde{m} \in \widetilde{D}$. On the other hand, since $\widetilde{N} \nsubseteq P \oplus \mathfrak{p}$, we deduce that $\pi_{1}(\tilde{N})+\pi_{2}(\widetilde{N}) M \nsubseteq P$. Hence by 2.3 ,

$$
(P: M) \subseteq(\widetilde{D}: \widetilde{M})=\left(P: \pi_{1}(\widetilde{N})+\pi_{2}(\widetilde{N}) M\right) \subseteq \sqrt{(P: M)},
$$

for $P$ is primary. So $\sqrt{(\widetilde{D}: \widetilde{M})}=\mathfrak{p}$, whence $\widetilde{D}$ is $\mathfrak{p}$-primary. The proof for primeness is similar.

Now assume that $P$ is weakly $\mathfrak{p}$-prime and $r_{1} r_{2} \widetilde{m} \in \widetilde{D}$. Thus for each $\widetilde{n} \in \widetilde{N}$, we have $r_{1} r_{2} \Delta(\widetilde{m}, \widetilde{n}) \in P$. By $P$ being weakly prime, we deduce that either $r_{1} \Delta(\widetilde{m}, \widetilde{n}) \in P$ or $r_{2} \Delta(\widetilde{m}, \widetilde{n}) \in P$. Therefore, either $\Delta(\widetilde{m}, \widetilde{N}) \subseteq\left(P:_{M} r_{1}\right)$ or $\Delta(\widetilde{m}, \widetilde{N}) \subseteq\left(P:_{M} r_{2}\right)$. Consequently, either $r_{1} \widetilde{m} \in \Delta^{P}(\widetilde{N})$ or $r_{2} \widetilde{m} \in \Delta^{P}(\widetilde{N})$ and $\widetilde{D}$ is weakly prime.

Under the conditions of the above result, in the case that $P$ is weakly $\mathfrak{p}$-prime, it may happen that $(\widetilde{D}: \widetilde{M}) \neq \mathfrak{p}$, as the following example shows.

Example 2.7. Let $M=R \oplus R$ and $P=\mathfrak{p} \oplus \mathfrak{q}$ for prime ideals $\mathfrak{p} \subset \mathfrak{q}$ of $R$. One can readily check that $P$ is weakly $\mathfrak{p}$-prime. Set $n=(0,1) \in M$ and $\widetilde{N}=R n \leq M \leq \widetilde{M}$. Then $\widetilde{D}=\Delta^{P}(\widetilde{N})=M \oplus \mathfrak{q}$ and $(\widetilde{D}: \widetilde{M})=\mathfrak{q}$.

\section{Prime submodules and radical of submodules}

In this section, assuming that $P$ is a prime submodule of $M$, we try to find exactly $\Delta^{P}(\tilde{N})$ for an arbitrary submodule $\tilde{N}$ of $\widetilde{M}$ and use it to present a formulation of $\operatorname{rad}(\tilde{N})$. Throughout this section $P$ is assumed to be a $\mathfrak{p}$-prime submodule of $M$. First we need the following well-known results (see for example [12]).

Lemma 3.1. Suppose $K \leq L \leq M, M^{\prime} \leq M, I \subseteq(K: M)$ and let $A^{\prime}$ be a submodule of an $R$-module $A$. Then

(a) $L$ is a $\mathfrak{p}$-prime submodule of $M$ if and only if $\frac{L}{K}$ is a $\frac{\mathfrak{p}}{I}$-prime $\frac{R}{I}$-submodule of $\frac{M}{K}$;

(b) the smallest $\mathfrak{p}$-prime submodule of $M$ containing $L$, if any exists, is $(L+\mathfrak{p} M)_{\mathfrak{p}}{ }^{c}$. If there is no such prime submodule, then $(L+\mathfrak{p} M)_{\mathfrak{p}}{ }^{c}=M$;

(c) $L$ is a $\mathfrak{p}$-prime submodule of $M$ if and only if $L=L_{\mathfrak{p}}{ }^{c}$ and $L_{\mathfrak{p}}$ is a $\mathfrak{p}_{\mathfrak{p}}$-prime submodule of $M_{\mathfrak{p}}$; 
(d) if $L$ is $\mathfrak{p}$-prime in $M$, then $L \cap M^{\prime}$ is either the whole $M^{\prime}$ or a $\mathfrak{p}$-prime submodule of $M^{\prime}$;

(e) every proper subspace of a vector space is 0-prime;

(f) $\operatorname{rad}_{M \oplus A}\left(M^{\prime} \oplus A^{\prime}\right)=\operatorname{rad}_{M}\left(M^{\prime}\right) \oplus \operatorname{rad}_{A}\left(A^{\prime}\right)$.

Now we have all the stuff needed to characterize $\Delta^{P}$ of submodules of $\widetilde{M}$.

Theorem 3.2. Assume that $P$ is a p-prime submodule of $M$ and $\widetilde{N} \leq \widetilde{M}$. Then

(a) if $\widetilde{N} \subseteq P \oplus \mathfrak{p}$, then $\Delta^{P}(\tilde{N})=\widetilde{M}$;

(b) if $\tilde{N} \nsubseteq P \oplus \mathfrak{p}$ but $\tilde{N} \subseteq M \oplus \mathfrak{p}$, then $\Delta^{P}(\tilde{N})=M \oplus \mathfrak{p}$;

(c) if $\widetilde{N} \nsubseteq M \oplus \mathfrak{p}$ and $\widetilde{N} \cap(M \oplus \mathfrak{p}) \nsubseteq P \oplus \mathfrak{p}$, then $\Delta^{P}(\widetilde{N})=P \oplus \mathfrak{p}$;

(d) otherwise $\Delta^{P}(\widetilde{N})=(\widetilde{N}+(P \oplus \mathfrak{p}))_{\mathfrak{p}}{ }^{c}$ is the smallest $\mathfrak{p}$-prime submodule $\widetilde{P}$ of $\widetilde{M}$ containing $\widetilde{N}$ with $\widetilde{P} \cap M=P$.

Proof. Case (a) follows from 2.1c and 2.1d. Now suppose that $\widetilde{N} \nsubseteq P \oplus \mathfrak{p}$ but $\widetilde{N} \subseteq M \oplus \mathfrak{p}$, then by $2.1 \mathrm{c}$ and $2.1 \mathrm{~d}$, we conclude that $M \oplus \mathfrak{p} \subseteq \widetilde{D}=\Delta^{P}(\widetilde{N})$. Also according to 2.6, $\widetilde{D}$ is a $\mathfrak{p}$-prime submodule of $\widetilde{M}$. But the only $\mathfrak{p}$-prime submodule of $\widetilde{M}$ containing $M \oplus \mathfrak{p}$ is $M \oplus \mathfrak{p}$.

Thus we can assume that $\widetilde{N} \nsubseteq M \oplus \mathfrak{p}$. Let $\widetilde{N}^{\prime}=\widetilde{N}+P \oplus \mathfrak{p}$. Note that by regularity $\tilde{N}^{\prime} \cap(M \oplus \mathfrak{p})=(\widetilde{N} \cap(M \oplus \mathfrak{p}))+P \oplus \mathfrak{p}$. Consequently, it follows from 2.4 and $3.1 \mathrm{~b}$ that any of the conditions or the results of (c) or (d) holds for $\widetilde{N}^{\prime}$ if and only if the same condition or result holds for $\widetilde{N}$. Therefore by replacing $\widetilde{N}$ with $\widetilde{N}^{\prime}$, we can assume that $P \oplus \mathfrak{p} \subseteq \tilde{N}$. Again by applying 2.4 and 3.1 a, and by passing to $\frac{R}{\mathfrak{p}}$ and $\frac{M}{P}$, we assume that $P=0=\mathfrak{p}$. In particular, $R$ is a domain and $M$ is torsion-free.

If case (c) holds, that is, $\widetilde{N} \nsubseteq M$ and $\widetilde{N} \cap M \neq 0$, then there is a $0 \neq m \in \widetilde{N} \cap M$ and $\widetilde{n} \in \widetilde{N}$ with $0 \neq r=\pi_{2}(\widetilde{n})$. Let $\widetilde{m} \in \widetilde{D}$, then $\Delta(m, \widetilde{m}) \in P=0$. Thus $\pi_{2}(\widetilde{m}) m=0$ and as $m \neq 0$ and $M$ is torsion-free, we deduce that $\pi_{2}(\widetilde{m})=0$. Moreover, $0=\Delta(\widetilde{n}, \widetilde{m})=$ $-r \pi_{1}(\widetilde{m})$, so $\pi_{1}(\widetilde{m})=0$ and hence $\widetilde{m}=0$ as required.

Finally assume that $\tilde{N} \nsubseteq M$ and $\tilde{N} \cap M=0$. As $\widetilde{N}_{0} \cap M_{0}=0$ (here $X_{0}$ means localization of $X$ on the zero ideal), $\widetilde{N}_{0}$ is a proper, and according to $3.1 \mathrm{e}$, a 0 -prime submodule of $\widetilde{M}_{0}$. Furthermore, by $3.1 \mathrm{~b} \widetilde{P}=\widetilde{N}_{0}{ }^{c}$ is the smallest 0-prime submodule containing $\widetilde{N}$. If $x \in \widetilde{P} \cap M$, then there is a $0 \neq r \in R$ such that $r x \in \widetilde{N} \cap M=0$. Since $M$ is torsion-free, we get $x=0$, that is, $\widetilde{P} \cap M=0=P$. This proves the second equality of $d$.

Now note that by 2.5 and $3.1 \mathrm{c}$ the first equality of (d) is equivalent to $\Delta_{M_{0}, R_{0}}^{0}\left(\widetilde{N}_{0}\right)=\widetilde{N}_{0}$. Thus by changing $R$ with $R_{0}$, we can assume that $R$ is a field and we just need to prove $\widetilde{D}=\Delta^{0}(\widetilde{N})=\widetilde{N}$. Assume that $\widetilde{m}=m+r \in \widetilde{M}$ and $\widetilde{n}_{1}=m_{1}+r_{1} \in \widetilde{N} \backslash M$. Thus $r_{1} \neq 0$. Now

$$
\widetilde{m} \in \Delta^{0}\left(\widetilde{n}_{1}\right) \Leftrightarrow r m_{1}-r_{1} m=\Delta\left(\widetilde{n}_{1}, \widetilde{m}\right)=0 \Leftrightarrow m=r \frac{m_{1}}{r_{1}} \Leftrightarrow m+r \in R\left(t_{\widetilde{n}_{1}}+1\right),
$$

where $t_{\widetilde{n}_{1}}=\frac{m_{1}}{r_{1}}$. Therefore as $\widetilde{N} \cap M=0$, we have $\widetilde{D}=\Delta^{0}(\widetilde{N})=\bigcap_{\widetilde{n} \in \widetilde{N} \backslash M} R\left(t_{\widetilde{n}}+1\right)$. Assume that $\widetilde{n}_{2}=m_{2}+r_{2} \in \widetilde{N} \backslash M$ such that $t_{\widetilde{n}_{1}} \neq t_{\widetilde{n}_{2}}$. Then $0 \neq r_{2} m_{1}-r_{1} m_{2}=$ $r_{2} \widetilde{n}_{1}-r_{1} \widetilde{n}_{2} \in \widetilde{N} \cap M$, a contradiction. It follows that there is a $t \in M$ such that for each $0 \neq \widetilde{n} \in \widetilde{N}, t=t_{\widetilde{n}}$ and $\widetilde{n}=\pi_{2}(\widetilde{n})(t+1)$. Thus in particular, $\widetilde{N} \subseteq \widetilde{D}=R(t+1)$. On the other hand since $\widetilde{n}_{1} \in \widetilde{N}$ and $r_{1} \neq 0$, we see that $t+1=\frac{1}{r_{1}} \widetilde{n} \in \widetilde{N}$, that is, $\widetilde{N}=\widetilde{D}$ and the proof is concluded.

As an application we get the following characterizations of prime submodules of $\widetilde{M}$. 
Corollary 3.3. For $\widetilde{N} \leq \widetilde{M}$ and a prime ideal $\mathfrak{p}$ of $R$ the following are equivalent.

(a) $\widetilde{N}$ is p-prime.

(b) $\tilde{N}=M \oplus \mathfrak{p}$ or $P=\widetilde{N} \cap M$ is $\mathfrak{p}$-prime in $M$ and either $\widetilde{N}=P \oplus \mathfrak{p}$ or $\Delta^{P}(\tilde{N})=\tilde{N}$.

(c) Either $\tilde{N}=M \oplus \mathfrak{p}$ or $\tilde{N}=P \oplus \mathfrak{p}$ or $\tilde{N}=\Delta^{P}(\widetilde{m})$ for some $\mathfrak{p}$-prime submodule $P$ of $M$ and an $\widetilde{m} \in \widetilde{M} \backslash(M \oplus \mathfrak{p})$.

Proof. (a) $\Rightarrow$ (b): Suppose $\widetilde{N} \neq M \oplus \mathfrak{p}$. Then by $3.1 \mathrm{~d}, P=\widetilde{N} \cap M$ is $\mathfrak{p}$-prime in $M$. Now since $(\widetilde{N}: \widetilde{M})=\mathfrak{p}$, we see that $\mathfrak{p} M \oplus \mathfrak{p} \subseteq \widetilde{N}$ and whence $\widetilde{N} \cap(M \oplus \mathfrak{p})=(\widetilde{N} \cap M) \oplus \mathfrak{p}=P \oplus \mathfrak{p}$. Thus cases (b) and (c) of 3.2 cannot occur. If case 3.2 a holds, then $\tilde{N}=P \oplus \mathfrak{p}$. Else according to $3.2 \mathrm{~d}, \Delta^{P}(\widetilde{N})=\widetilde{N}_{\mathfrak{p}}{ }^{c}=\widetilde{N}$ by $3.1 \mathrm{c}$.

(b) $\Rightarrow$ (c): Assume that neither $\widetilde{N}=P \oplus \mathfrak{p}$ nor $\tilde{N}=M \oplus \mathfrak{p}$. Then (b) says that $\widetilde{N}=\Delta^{P}(\widetilde{N})$ for some $\mathfrak{p}$-prime submodule $P$ of $M$. Clearly cases (a)-(c) of 3.2 cannot happen. Thus $\tilde{N} \nsubseteq M \oplus \mathfrak{p}$. Let $\widetilde{m} \in \widetilde{N} \backslash(M \oplus \mathfrak{p})$. If $r \widetilde{m} \in M \oplus \mathfrak{p}$, then $r \pi_{2}(\widetilde{m}) \in \mathfrak{p}$ and as $\pi_{2}(\widetilde{m}) \notin \mathfrak{p}$, we should have $r \in \mathfrak{p}$. So $r \pi_{1}(\widetilde{m}) \in \mathfrak{p} M \subseteq P$. Hence $R \widetilde{m} \cap(M \oplus \mathfrak{p}) \subseteq P \oplus \mathfrak{p}$ and $R \widetilde{m}$ satisfies the conditions of $3.2 \mathrm{~d}$ and

$$
\Delta^{P}(\widetilde{m})=\Delta^{P}(R \widetilde{m})=(R \widetilde{m}+P \oplus \mathfrak{p})_{\mathfrak{p}}{ }^{c} \subseteq(\widetilde{N}+P \oplus \mathfrak{p})_{\mathfrak{p}}{ }^{c}=\Delta^{P}(\tilde{N}) .
$$

On the other hand, $R \widetilde{m} \subseteq \widetilde{N}$ and according to $2.1 \mathrm{c}, \Delta^{P}(\tilde{N}) \subseteq \Delta^{P}(\widetilde{m})$. Consequently, $\widetilde{N}=\Delta^{P}(\widetilde{N})=\Delta^{P}(\widetilde{m})$.

(c) $\Rightarrow$ (a): If $\widetilde{N}=P \oplus \mathfrak{p}$ or $\widetilde{N}=M \oplus \mathfrak{p}$, then the result is obvious. Assume $\widetilde{N}=\Delta^{P}(\widetilde{m})$ for a p-prime submodule $P$ of $M$ and an $\widetilde{m} \in \widetilde{M} \backslash(M \oplus \mathfrak{p})$. Clearly cases (a) and (b) of 3.2 do not occur for $R \widetilde{m}$ and in either of the cases (c) or (d) of the previous theorem, $\Delta^{P}(R \widetilde{m})$ is a $\mathfrak{p}$-prime submodule of $\widetilde{M}$, as required.

Using this corollary we can inductively get a characterization of prime submodules of finitely generated free modules. For this first we need some notations. Note that the $R$-module $\widetilde{M}$ can also be considered as a commutative ring by defining $\mathrm{mm}^{\prime}=0$ for all $m, m^{\prime} \in M$ (this ring is usually called the idealization of $M$ ). Thus we can compute determinants of square matrices with entries in $\widetilde{M}$.

Notation 3.4. Suppose that

$$
\mathbf{A}=\left(\begin{array}{ccc}
a_{1,1} & \ldots & a_{1, n} \\
\vdots & \ddots & \vdots \\
a_{k, 1} & \ldots & a_{k, n}
\end{array}\right)
$$

is a $k \times n$ matrix with $k \leq n$ and entries in $R$. Let $M=R^{n-k+1}$. If in each row of $\mathbf{A}$ we consider the first $n-k+1$ entries as an element of $M \leq \widetilde{M}$ and the other entries of the row as elements of $R \leq \widetilde{M}$, then we denote the determinant of $\mathbf{A}$ by $\operatorname{det}_{n-k+1}(\mathbf{A})$ which is an element of $R^{n-k+1}$.

Example 3.5. Let $\mathbf{A}=\left(\begin{array}{ccc}1 & 2 & 5 \\ -3 & 4 & 2\end{array}\right)$ over $\mathbb{Z}$. Then $\operatorname{det}_{2}(\mathbf{A})=(1,2) 2-5(-3,4)=$ $(17,-16) \in \mathbb{Z}^{2}$.

In what follows, we consider two submodules $A$ and $B$ of $R^{n}$ the same, up to a permutation of coordinates, if there exists a permutation $\sigma \in S_{n}$, such that $\left(x_{1}, \ldots, x_{n}\right) \in A \Leftrightarrow$ $\left(x_{\sigma(1)}, \ldots, x_{\sigma(n)}\right) \in B$. If $I$ is an ideal of $R$, by $I^{n}$ we mean the submodule $\underbrace{I \times I \times \cdots \times I}_{n \text { times }}$ of $R^{n}$. 
Theorem 3.6. Assume that $\mathfrak{p}$ is a prime ideal of $R$ and $\widetilde{P}<R^{n}$. Then $\widetilde{P}$ is a $\mathfrak{p}$-prime submodule of $R^{n}$ if and only if there exist an integer $0 \leq k<n$ and $a_{i, j} \in R$ with $1 \leq i \leq k$ and $1 \leq j \leq n-i+1$, such that $a_{i, n-i+1} \notin \mathfrak{p}$ and up to a permutation of coordinates $\widetilde{P}=\left\{\left(x_{1}, \ldots, x_{n}\right) \mid \operatorname{det}_{n-k}\left(\mathbf{A}\left(x_{1}, \ldots, x_{n}\right)\right) \in \mathfrak{p}^{n-k}\right\}$, where

$$
\mathbf{A}\left(x_{1}, \ldots, x_{n}\right)=\left(\begin{array}{cccccc}
x_{1} & \ldots & \ldots & \ldots & \ldots & x_{n} \\
a_{1,1} & \ldots & \ldots & \ldots & \ldots & a_{1, n} \\
a_{2,1} & \ldots & \ldots & \ldots & a_{2, n-1} & 0 \\
a_{3,1} & \ldots & \ldots & a_{3, n-2} & 0 & 0 \\
\vdots & \ddots & \vdots & \vdots & \vdots & \vdots \\
a_{k, 1} & \ldots & a_{k, n-k+1} & 0 & \ldots & 0
\end{array}\right) .
$$

Before stating the proof, it should be noted that in the case $k=0$, we have $\operatorname{det}_{n-k}\left(\mathbf{A}\left(x_{1}, \ldots, x_{n}\right)\right)=\left(x_{1}, \ldots, x_{n}\right)$ and hence the condition in this case holds if and only if $\widetilde{P}=\mathfrak{p}^{n}$.

Proof. $(\Rightarrow)$ : We prove the result by induction on $n$. If $n=1$, then $\widetilde{P}=\mathfrak{p}$ and the result holds with $k=0$, according to the above remark. Assume $n>1$. If $\widetilde{P}=\mathfrak{p}^{n}$, then again the result holds by the above note. Thus we assume that an entry of an element of $\widetilde{P}$ is not in $\mathfrak{p}$. Since we are working up to a permutation of coordinates, we assume that this entry is on the last coordinate. Hence in part c of 3.3 (with $M=R^{n-1}$ and $\widetilde{N}=\widetilde{P}$ ) the first two cases cannot happen. Therefore, we have $\widetilde{P}=\Delta^{P}(\widetilde{m})$ for some $\mathfrak{p}$-prime submodule $P$ of $R^{n-1}$ and an $\widetilde{m} \in R^{n} \backslash\left(R^{n-1} \oplus \mathfrak{p}\right)$. Suppose that $\widetilde{m}=\left(a_{1,1}, \ldots, a_{1, n}\right)$. Then $a_{1, n} \notin \mathfrak{p}$.

By induction hypothesis, up to a permutation of coordinates $P=$ $\left\{\left(x_{1}, \ldots, x_{n-1}\right) \mid \operatorname{det}_{n-k}\left(\mathbf{B}\left(x_{1}, \ldots, x_{n-1}\right)\right) \in \mathfrak{p}^{n-k}\right\}$, where

$$
\mathbf{B}\left(x_{1}, \ldots, x_{n-1}\right)=\left(\begin{array}{cccccc}
x_{1} & \ldots & \ldots & \ldots & \ldots & x_{n-1} \\
a_{2,1} & \ldots & \ldots & \ldots & \ldots & a_{2, n-1} \\
a_{3,1} & \ldots & \ldots & \ldots & a_{3, n-2} & 0 \\
\vdots & \ddots & \vdots & \vdots & \vdots & \vdots \\
a_{k, 1} & \ldots & a_{k, n-k+1} & 0 & \ldots & 0
\end{array}\right),
$$

for suitable $k$ and $a_{i, j}$ (note that we have started the first indices of $a_{i, j}$ 's in $\mathbf{B}$ from 2 instead of 1). Now:

$$
\begin{aligned}
& \left(x_{1}, \ldots, x_{n}\right) \in \widetilde{P}=\Delta^{P}(\widetilde{m}) \\
\Leftrightarrow & m:=x_{n}\left(a_{1,1}, \ldots, a_{1, n-1}\right)-a_{1, n}\left(x_{1}, \ldots, x_{n-1}\right) \in P \\
\Leftrightarrow & \operatorname{det}_{n-k}(\mathbf{B}(m)) \in \mathfrak{p}^{n-k} \\
\Leftrightarrow & x_{n} \operatorname{det}_{n-k}\left(\mathbf{B}\left(a_{1,1}, \ldots, a_{1, n-1}\right)\right)-a_{1, n} \operatorname{det}_{n-k}\left(\mathbf{B}\left(x_{1}, \ldots, x_{n-1}\right)\right) \in \mathfrak{p}^{n-k} \\
\Leftrightarrow & \operatorname{det}_{n-k}\left(\mathbf{A}\left(x_{1}, \ldots, x_{n}\right)\right) \in \mathfrak{p}^{n-k},
\end{aligned}
$$

where the last equivalency holds by expanding $\operatorname{det}_{n-k}\left(\mathbf{A}\left(x_{1}, \ldots, x_{n}\right)\right)$.

$(\Leftarrow)$ : We use induction on $n$. If $n=1$, then $k=0$ and $\widetilde{P}=\mathfrak{p}$ is a prime submodule of $R$. Assume $n>1$. For $\left(x_{1}, \ldots, x_{n-1}\right) \in R^{n-1}$, let $B\left(x_{1}, \ldots, x_{n-1}\right)$ be defined by $*$. Set $P=\left\{\left(x_{1}, \ldots, x_{n-1}\right) \mid \operatorname{det}_{n-k}\left(\mathbf{B}\left(x_{1}, \ldots, x_{n-1}\right)\right) \in \mathfrak{p}^{n-k}\right\}$ which is a prime submodule of $M=R^{n-1}$ by induction hypothesis. Now $* *$ shows that $\widetilde{P}=\Delta^{P}(\widetilde{m})$ where $\widetilde{m}=$ $\left(a_{1,1}, \ldots, a_{1, n}\right)$. Note that since $a_{1, n} \notin \mathfrak{p}$, we have $\widetilde{m} \in \widetilde{M} \backslash(M \oplus \mathfrak{p})$. Thus by 3.3c, $\widetilde{P}$ is a prime submodule of $\widetilde{M}=R^{n}$.

The above theorem should be compared with [15, Theorem 1.6], which presents a characterization of prime submodules of $R^{n}$ using determinants of some matrices. In Theorem 1.6 of [15], for checking if a submodule of $R^{n}$ is prime, one should consider all $k$-minors 
of a certain matrix. But in Theorem 3.6, we just need to find one determinant. Also the matrix in 3.6, has a simpler form (it has many zeros) than the matrix in [15].

Next we are going to present a formulation for $\operatorname{rad}(\widetilde{N})$ where $\widetilde{N}$ is an arbitrary submodule of $\widetilde{M}$. For this we need the following lemma.

Lemma 3.7. Assume that $\mathfrak{p}$ is a prime ideal of $R$ and $\widetilde{N} \leq \widetilde{M}$ is such that $\widetilde{N} \nsubseteq M \oplus \mathfrak{p}$ and set $N_{1}=\widetilde{N} \cap M$, and $N_{2}=\pi_{1}(\widetilde{N} \cap(M \oplus \mathfrak{p}))$. Then $\left(N_{1}+\mathfrak{p} M\right)_{\mathfrak{p}}{ }^{c}=\left(N_{2}+\mathfrak{p} M\right)_{\mathfrak{p}}{ }^{c}$.

Proof. ( $\subseteq$ ): It is satisfied since $N_{1} \subseteq N_{2}$.

(): We just need to show that $N_{2} \subseteq\left(N_{1}+\mathfrak{p} M\right)_{\mathfrak{p}}{ }^{c}$. Let $n_{2} \in N_{2}$. Then by definition of $N_{2}$, there should exist an $\widetilde{n} \in \widetilde{N} \cap(M \oplus \mathfrak{p})$ such that $\widetilde{n}=n_{2}+r$ for some $r \in R$. Thus $r \in \mathfrak{p}$. By assumption there are $s \in R \backslash \mathfrak{p}$ and $m \in M$ with $m+s \in \tilde{N}$. Now $x=s\left(n_{2}+r\right)-r(m+s)=s n_{2}-r m \in \widetilde{N} \cap M=N_{1}$ and $s n_{2}=x+r m \in N_{1}+\mathfrak{p} M$. Consequently, $n_{2} \in\left(N_{1}+\mathfrak{p} M\right)_{\mathfrak{p}}{ }^{c}$, and the result follows.

Theorem 3.8. Suppose that $\widetilde{N} \leq \widetilde{M}$ and set $N_{1}=\widetilde{N} \cap M$. Then

$$
\operatorname{rad}_{\widetilde{M}}(\tilde{N})=\left(\operatorname{rad}_{M}\left(\pi_{1}(\tilde{N})\right) \oplus \sqrt{\pi_{2}(\tilde{N})}\right) \cap \Delta^{\operatorname{rad}_{M}\left(N_{1}\right)}(\tilde{N}) .
$$

Proof. $(\subseteq)$ : By 3.1f, we have $\operatorname{rad}_{\widetilde{M}}(\tilde{N}) \subseteq \operatorname{rad}_{M}\left(\pi_{1}(\tilde{N})\right) \oplus \sqrt{\pi_{2}(\tilde{N})}$, because $\tilde{N} \subseteq \pi_{1}(\tilde{N}) \oplus$ $\pi_{2}(\tilde{N})$. According to $3.1 \mathrm{~b}, \operatorname{rad}_{M}\left(N_{1}\right)=\bigcap_{\mathfrak{p} \in \operatorname{spec}(R)}\left(N_{1}+\mathfrak{p} M\right)_{\mathfrak{p}}{ }^{c}$, where $\operatorname{spec}(R)$ denotes the set of prime ideals of $R$. Therefore by $2.1 \mathrm{f}$,

$$
\Delta^{\operatorname{rad}_{M}\left(N_{1}\right)}(\widetilde{N})=\bigcap_{\mathfrak{p} \in \operatorname{spec}(R)} \Delta^{\left(N_{1}+\mathfrak{p} M\right)_{\mathfrak{p}}{ }^{c}(\tilde{N})} .
$$

Thus if $\mathfrak{p} \in \operatorname{spec}(R)$ and $P=\left(N_{1}+\mathfrak{p} M\right)_{\mathfrak{p}}{ }^{c}$, we just need to show that $\operatorname{rad}_{\widetilde{M}}(\tilde{N}) \subseteq \Delta^{P}(\tilde{N})$. If $P=M$ we get $\Delta^{P}(\widetilde{N})=\widetilde{M} \supseteq \operatorname{rad}_{\widetilde{M}}(\widetilde{N})$. So assume $P \neq M$, hence $P$ is a p-prime submodule of $M$, by 3.1 b. If $\widetilde{N} \subseteq M \oplus \mathfrak{p}$, then by $3.2, \Delta^{P}(\widetilde{N})$ is either $M \oplus \mathfrak{p}$ or $\widetilde{M}$. In both cases clearly $\operatorname{rad}_{\widetilde{M}}(\widetilde{N}) \subseteq \Delta^{P}(\widetilde{N})$.

Thus we can assume that $\widetilde{N} \nsubseteq M \oplus \mathfrak{p}$. According to previous lemma, if $N_{2}=\pi_{1}(\tilde{N} \cap(M \oplus$ $\mathfrak{p})$ ), then $P=\left(N_{2}+\mathfrak{p} M\right)_{\mathfrak{p}}{ }^{c}$, in particular, $N_{2} \subseteq P$. Hence $\tilde{N} \cap(M \oplus \mathfrak{p}) \subseteq N_{2} \oplus \mathfrak{p} \subseteq P \oplus \mathfrak{p}$. So by $3.2, \Delta^{P}(\widetilde{N})$ is a p-prime submodule containing $\widetilde{N}$ and hence $\operatorname{rad}_{\widetilde{M}}(\widetilde{N})$, as required.

$(\supseteq)$ : Let $\mathfrak{p}$ be an arbitrary prime ideal of $R$ and set $\widetilde{P}=(\widetilde{N}+\mathfrak{p} \widetilde{M})_{\mathfrak{p}}{ }^{c}$. We just need to show that the right hand side of the claimed equality is contained in $\widetilde{P}$. If $\widetilde{P}=P \oplus I$ where $I$ is either $R$ or $\mathfrak{p}$ and $P$ is either $M$ or a $\mathfrak{p}$-prime submodule of $M$, then as $\widetilde{N} \subseteq \widetilde{P}$ we get $\pi_{1}(\tilde{N}) \subseteq P$ and $\pi_{2}(\widetilde{N}) \subseteq I$. Therefore $\operatorname{rad}_{M}\left(\pi_{1}(\tilde{N})\right) \oplus \sqrt{\pi_{2}(\widetilde{N})} \subseteq \widetilde{P}$, as required. Thus we assume that $\widetilde{P}$ is not in the form mentioned above.

Set $N_{2}=\pi_{1}(\tilde{N} \cap(M \oplus \mathfrak{p}))$ and $P=\left(N_{2}+\mathfrak{p} M\right)_{\mathfrak{p}}{ }^{c}$. If $n_{2} \in N_{2}$, then for some $r \in \mathfrak{p}$ we have $n_{2}+r \in \widetilde{N}$. So $n_{2} \in \widetilde{N}+\mathfrak{p} \widetilde{M}$. Therefore, $\left(N_{2}+\mathfrak{p} M\right) \oplus \mathfrak{p} \subseteq \widetilde{N}+\mathfrak{p} \widetilde{M}$, whence $P \oplus \mathfrak{p}=\left(\left(N_{2}+\mathfrak{p} M\right) \oplus \mathfrak{p}\right)_{\mathfrak{p}}{ }^{c} \subseteq(\widetilde{N}+\mathfrak{p} \widetilde{M})_{\mathfrak{p}}{ }^{c}=\widetilde{P}$. Thus if $P=M$, then $\widetilde{P}$ should be either $M \oplus R$ or $M \oplus \mathfrak{p}$, against what we assumed above. Hence $P$ is a $\mathfrak{p}$-prime submodule of $M$. Also if $\widetilde{N} \subseteq P \oplus \mathfrak{p}$, then the $\mathfrak{p}$-prime submodule $P \oplus \mathfrak{p}$ should contain $\widetilde{P}$ which is the smallest $\mathfrak{p}$-prime submodule of $\widetilde{M}$ containing $\widetilde{N}$. Therefore, $\widetilde{P}=P \oplus \mathfrak{p}$, again contradicting our assumption. Moreover, $\tilde{N} \cap(M \oplus \mathfrak{p}) \subseteq N_{2} \oplus \mathfrak{p} \subseteq P \oplus \mathfrak{p}$. Consequently, we see that $P$ and $\widetilde{N}$ satisfy the conditions of case d of 3.2. It follows that

$$
\widetilde{P}=(\widetilde{N}+\mathfrak{p} \widetilde{M})_{\mathfrak{p}}{ }^{c} \subseteq \Delta^{P}(N)=(\widetilde{N}+(P \oplus \mathfrak{p}))_{\mathfrak{p}}{ }^{c} \subseteq \widetilde{P}_{\mathfrak{p}}{ }^{c}=\widetilde{P},
$$

that is, $\widetilde{P}=\Delta^{P}(\tilde{N})$. Because $\widetilde{N}$ satisfies case $\mathrm{d}$ of 3.2 , we deduce that $\widetilde{N} \nsubseteq M \oplus \mathfrak{p}$. Therefore according to $3.7, P=\left(N_{1}+\mathfrak{p} M\right)_{\mathfrak{p}}{ }^{c} \supseteq \operatorname{rad}\left(N_{1}\right)$ and $\widetilde{P}=\Delta^{P}(\widetilde{N}) \supseteq \Delta^{\operatorname{rad}\left(N_{1}\right)}(\widetilde{N})$, as claimed. 
The following example shows how we can apply 3.8 .

Example 3.9. Assume that $M=R=\mathbb{Z}$ and $\tilde{N}=\mathbb{Z}(2,2)+\mathbb{Z}(3,0)$. Then $\pi_{1}(\tilde{N})=\mathbb{Z}$, $\pi_{2}(\tilde{N})=2 \mathbb{Z}$ and $N_{1}=\tilde{N} \cap M=\tilde{N} \cap(\mathbb{Z} \oplus 0)=3 \mathbb{Z}$. By definition

$$
\begin{aligned}
\Delta^{3 \mathbb{Z}}(\widetilde{N}) & =\Delta^{3 \mathbb{Z}}(2,2) \cap \Delta^{3 \mathbb{Z}}(3,0) \\
& =\left\{\left(n_{1}, n_{2}\right) \mid 2 n_{1}-2 n_{2} \in 3 \mathbb{Z}\right\} \cap\left\{\left(n_{1}, n_{2}\right) \mid 3 n_{2} \in 3 \mathbb{Z}\right\} \\
& =\left\{\left(n_{1}, n_{2}\right) \mid n_{1}-n_{2} \in 3 \mathbb{Z}\right\} .
\end{aligned}
$$

Therefore according to 3.8 ,

$$
\begin{aligned}
\operatorname{rad}(\tilde{N}) & =(\sqrt{\mathbb{Z}} \oplus \sqrt{2 \widetilde{Z}}) \cap \Delta^{3 \mathbb{Z}}(\tilde{N})=(\mathbb{Z} \oplus 2 \mathbb{Z}) \cap\left\{\left(n_{1}, n_{2}\right) \mid n_{1}-n_{2} \in 3 \mathbb{Z}\right\} \\
& =\{(3 t+2 k, 2 k) \mid t, k \in \mathbb{Z}\}=\tilde{N},
\end{aligned}
$$

that is, $\widetilde{N}$ is a radical submodule of $\widetilde{M}$.

\section{Delta operation and primary decompositions}

At this final section we pay some attention to primary decompositions of submodules and their behavior under the delta operation. Recall that if $A=\bigcap_{i=1}^{n} Q_{i}$ is a minimal primary decomposition of a submodule $A$ of $M$, then $\operatorname{Ass}(\mathrm{A})=\left\{\sqrt{\left(\mathrm{Q}_{\mathrm{i}}: \mathrm{M}\right)}\right\}_{\mathrm{i}=1}^{\mathrm{n}}$ and $\min (A)$ is the set of minimal elements of $\operatorname{Ass}(\mathrm{A})$.

Theorem 4.1. Suppose that $A=\bigcap_{i=1}^{n} Q_{i}$ is a minimal primary decomposition and $\widetilde{A}=$ $\Delta^{A}(\widetilde{N})$ for some submodule $\widetilde{N}$ of $\widetilde{M}$ such that $\tilde{N} \nsubseteq Q_{i} \oplus\left(Q_{i}: M\right)$ for each $i$. Then $\widetilde{A}=\bigcap_{i=1}^{n} \Delta^{Q_{i}}(\widetilde{N})$ is a primary decomposition of $\widetilde{A}$ and $\operatorname{Ass}(\widetilde{\mathrm{A}}) \subseteq \operatorname{Ass}(\mathrm{A})$. Moreover $\min (\widetilde{A})=\min (A)$ and if $\widetilde{N} \nsubseteq M \oplus \mathfrak{p}$ for each embedded prime $\mathfrak{p}$ of $A$, then this primary decomposition of $\widetilde{A}$ is minimal.

Proof. The first statement follows from 2.1f and 2.6. Suppose that this primary decomposition of $\widetilde{A}$ is not minimal and let $\mathfrak{p}_{i}=\sqrt{\left(Q_{i}: M\right)}$. Then for some $1 \leq i \leq n$, we should have $\bigcap_{i \neq j=1}^{n} \Delta^{Q_{j}}(\widetilde{N}) \subseteq \Delta^{Q_{i}}(\widetilde{N})$. Then $\left(\bigcap_{i \neq j=1}^{n} Q_{j}\right) \oplus 0 \subseteq \bigcap_{i \neq j=1}^{n}\left(Q_{j} \oplus\left(Q_{j}: M\right)\right) \subseteq$ $\Delta^{Q_{i}}(\tilde{N})$ by 2.4. Hence $\pi_{2}(\tilde{N})\left(\bigcap_{i \neq j=1}^{n} Q_{j}\right)=\Delta\left(\left(\cap_{i \neq j=1}^{n} Q_{j}\right) \oplus 0, \widetilde{N}\right) \subseteq Q_{i}$. Because of the minimality of the primary decomposition of $A$ and the fact that $Q_{i}$ is $\mathfrak{p}_{i}$-primary, we deduce that $\pi_{2}(\widetilde{N}) \subseteq \mathfrak{p}_{i}$, that is, $\widetilde{N} \subseteq M \oplus \mathfrak{p}_{i}$. On the other hand,

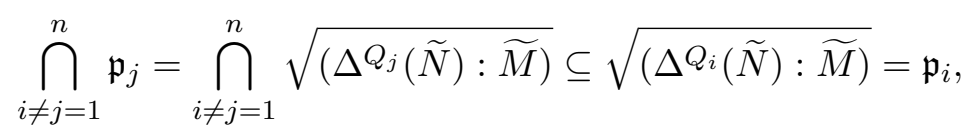

where the flanking equalities follow from 2.6. Consequently $\mathfrak{p}_{j} \subseteq \mathfrak{p}_{i}$ for some $j \neq i$ and $\mathfrak{p}_{i}$ is an embedded prime of $A$, and the second statement is established.

The above theorem proposes the question "when a submodule $\widetilde{A}$ of $\widetilde{M}$ is of the form $\Delta^{B}(\widetilde{N})$ for some submodules $B$ and $\widetilde{N}$ of $M$ and $\widetilde{M}$, respectively?" Regarding this, we have:

Proposition 4.2. Suppose that $\widetilde{N}=\Delta^{A}(\widetilde{K})$ for some $\widetilde{K} \leq \widetilde{M}$ and $\left(A:_{M} \pi_{2}(\widetilde{N})\right)=A$ (for example, if $\pi_{2}(\widetilde{N}) \nsubseteq Z\left(\frac{M}{A}\right)=\{r \in R \mid \exists m \in M \backslash A$ : rm $\in A\}$ ). Then $\widetilde{N}=\Delta^{B}(\widetilde{N})$ where $B=\Delta(\tilde{N}, \widetilde{N})$.

Proof. Let $B^{\prime}=\left(A:_{M} \pi_{2}\left(\Delta^{A}(\tilde{N})\right)\right)$. First we show that $\Delta^{A}\left(\Delta^{A}(\tilde{N})\right)=\Delta^{B^{\prime}}(\tilde{N})$. In the following for any element $\widetilde{m} \in \widetilde{M}$ we denote $\pi_{i}(\widetilde{m})$ by $m_{i}(i=1,2)$. 
$(\subseteq)$ : Assume that $\widetilde{x} \in \Delta^{A}\left(\Delta^{A}(\widetilde{N})\right), \widetilde{n} \in \widetilde{N}$ and $\widetilde{a} \in \Delta^{A}(\widetilde{N})$. Then $z=a_{1} n_{2}-a_{2} n_{1}=$ $\Delta(\widetilde{a}, \widetilde{n}) \in A$ and $\Delta(\widetilde{a}, \widetilde{x}) \in A$. Thus

$$
\begin{aligned}
a_{2} \Delta(\widetilde{n}, \widetilde{x}) & =a_{2}\left(n_{1} x_{2}-n_{2} x_{1}\right)=\left(a_{2} n_{1}\right) x_{2}-a_{2} n_{2} x_{1} \\
& =\left(a_{1} n_{2}-z\right) x_{2}-a_{2} n_{2} x_{1}=n_{2}\left(a_{1} x_{2}-a_{2} x_{1}\right)-z x_{2} \\
& =n_{2} \Delta(\widetilde{a}, \widetilde{x})-z x_{2} \in A,
\end{aligned}
$$

that is, $\Delta(\widetilde{n}, \widetilde{x}) \in\left(A:_{M} a_{2}\right)$. Since $\widetilde{a} \in \Delta^{A}(\widetilde{N})$ was arbitrary, we conclude that $\Delta(\widetilde{n}, \widetilde{x}) \in$ $\left(A: M \pi_{2}\left(\Delta^{A}(\widetilde{N})\right)\right)=B^{\prime}$, and because $\widetilde{n}$ was arbitrary we deduce $\widetilde{x} \in \Delta^{B^{\prime}}(\widetilde{N})$ and hence $\Delta^{A}\left(\Delta^{A}(\tilde{N})\right) \subseteq \Delta^{B^{\prime}}(\tilde{N})$.

$(\supseteq)$ : Let $\widetilde{x} \in \Delta^{B^{\prime}}(\widetilde{N}), \widetilde{n} \in \widetilde{N}$ and $\widetilde{a} \in \Delta^{A}(\widetilde{N})$. Then $z=a_{1} n_{2}-n_{1} a_{2} \in A$ and $\Delta(\widetilde{n}, \widetilde{x}) \in A$

$$
\begin{aligned}
n_{2} \Delta(\widetilde{a}, \widetilde{x}) & =\left(n_{2} a_{1}\right) x_{2}-n_{2} a_{2} x_{1}=\left(a_{2} n_{1}+z\right) x_{2}-n_{2} a_{2} x_{1} \\
& =a_{2}\left(n_{1} x_{2}-n_{2} x_{1}\right)+z x_{2}=a_{2} \Delta(\widetilde{n}, \widetilde{x})+z x_{2} \in A,
\end{aligned}
$$

which similarly to the $(\subseteq)$ case, gives $\widetilde{x} \in \Delta^{\left(A_{M_{M}} \pi_{2}(\widetilde{N})\right)}\left(\Delta^{A}(\widetilde{N})\right)=\Delta^{A}\left(\Delta^{A}(\widetilde{N})\right)$ according to the assumption of the theorem. So $\Delta^{B^{\prime}}(\widetilde{N}) \subseteq \Delta^{A}\left(\Delta^{A}(\widetilde{N})\right)$.

Now note that since $\widetilde{N}=\Delta^{A}(\widetilde{K})$ for some $\widetilde{K} \leq \widetilde{M}, \widetilde{N}=\Delta^{A}\left(\Delta^{A}(\widetilde{N})\right)=\Delta^{B^{\prime}}(\widetilde{N})$ according to 2.2. In particular, $B=\Delta(\widetilde{N}, \widetilde{N}) \subseteq B^{\prime}$. Consequently, $\widetilde{N} \subseteq \Delta^{B}(\widetilde{N}) \subseteq$ $\Delta^{B^{\prime}}(\widetilde{N})=\widetilde{N}$, and hence $\widetilde{N}=\Delta^{B}(\widetilde{N})$.

This suggests to search for submodules $\widetilde{N}$ of $\widetilde{M}$ with $\widetilde{N}=\Delta^{B}(\widetilde{N})$ with $B=\Delta(\widetilde{N}, \widetilde{N})$.

Theorem 4.3. Suppose that $M$ is torsion-free and $B=\Delta(\tilde{N}, \tilde{N}) \neq 0$ and is cyclic. Then $\widetilde{N}=\Delta^{B}(\widetilde{N})$. In particular, if $B$ is proper and has a minimal primary decomposition $B=\bigcap_{i=1}^{n} Q_{i}$, then $\widetilde{N}=\bigcap_{i=1}^{n} \Delta^{Q_{i}}(\widetilde{N})$ is a primary decomposition of $\widetilde{N}$.

Proof. By assumption $B=R d$, where $0 \neq d=\sum_{i=1}^{k} a_{i} \alpha_{i}$ with $a_{i} \in R, 0 \neq \alpha_{i}=\Delta\left(\widetilde{m}_{i}, \widetilde{n}_{i}\right)$ and $\widetilde{m}_{i}, \widetilde{n}_{i} \in \widetilde{N}$. In particular as $\alpha_{i} \in B$, there exists $s_{i} \in R$, with $\alpha_{i}=s_{i} d$. Thus $d=\left(\sum_{i=1}^{k} a_{i} s_{i}\right) d$ and since $M$ is torsion-free we get $\sum_{i=1}^{k} a_{i} s_{i}=1 \quad(*)$. By definition of $B$, it is obvious that $\widetilde{N} \subseteq \Delta^{B}(\widetilde{N})$. For the converse inclusion, let $\widetilde{x} \in \Delta^{B}(\widetilde{N})$ be arbitrary. Then $\Delta\left(\widetilde{x}, \widetilde{m}_{i}\right), \Delta\left(\widetilde{x}, \widetilde{n}_{i}\right) \in B=R d$, that is, there are $r_{i}, r_{i}^{\prime} \in R$ such that for each $i$ :

$$
\left\{\begin{array}{ll}
m_{i 2} x_{1}-m_{i 1} x_{2}=r_{i} d & (1) \\
n_{i 2} x_{1}-n_{i 1} x_{2}=r_{i}^{\prime} d & (2)
\end{array},\right.
$$

where for any $j=1,2$ and $\widetilde{z} \in \widetilde{M}$ we have set $z_{j}=\pi_{j}(\widetilde{z})$. Now if we set $c_{i}=n_{i 2} r_{i}-m_{i 2} r_{i}^{\prime}$, then by subtracting $m_{i 2}$ times Eq. (2) from $n_{i 2}$ times Eq. (1) it follows that $-\alpha_{i} x_{2}=c_{i} d$. Hence $c_{i} \alpha_{i}=c_{i} s_{i} d=-s_{i} x_{2} \alpha_{i}$ and so by torsion-freeness, $c_{i}=-s_{i} x_{2} \quad(* *)$. Also $d x_{2}=$ $\sum_{i=1}^{k} a_{i} \alpha_{i} x_{2}=-\sum_{i=1}^{k} a_{i} c_{i} d$. Therefore,

$$
x_{2}=-\sum_{i=1}^{k} a_{i} c_{i}=\sum_{i=1}^{k}\left(a_{i} r_{i}^{\prime} m_{i 2}-a_{i} r_{i} n_{i 2}\right)
$$

Let $1 \leq i \leq k$ be such that $m_{i 2} \neq 0$. Then from (1) we deduce that $s_{i} m_{i 2} x_{1}=$ $s_{i} r_{i} d+s_{i} m_{i 1} x_{2}=r_{i} \alpha_{i}-c_{i} m_{i 1}$ (by (**)). Replacing $c_{i}$ and $\alpha_{i}$ with their definitions, we get that $s_{i} m_{i 2} x_{1}=m_{i 2} m_{i 1} r_{i}^{\prime}-r_{i} m_{i 2} n_{i 1}$. Cancelling out $m_{i 2}$ 's, we conclude $s_{i} x_{1}=r_{i}^{\prime} m_{i 1}-r_{i} n_{i 1}$. If $i$ is such that $m_{i 2}=0$, then $n_{i 2} \neq 0$ (else $\alpha_{i}=0$ ), so again we deduce the same equation for $s_{i} x_{1}$, using Eq. (2) instead of (1). Now summing up over all $i$ 's and using (*) we see that

$$
x_{1}=\sum_{i=1}^{k} a_{i} s_{i} x_{1}=\sum_{i=1}^{k}\left(a_{i} r_{i}^{\prime} m_{i 1}-a_{i} r_{i} n_{i 1}\right)
$$


Adding Eq. (3) and (4) we finally get $\widetilde{x}=x_{1}+x_{2}=\sum_{i=1}^{k}\left(a_{i} r_{i}^{\prime} \widetilde{m}_{i}-a_{i} r_{i} \widetilde{n}_{i}\right) \in \widetilde{N}$, as required.

For the "in particular" statement, by 4.1, we just need to show that $\widetilde{N} \nsubseteq Q_{i} \oplus\left(Q_{i}: M\right)$ for each $1 \leq i \leq n$. Suppose that for $i \leq t$ we have $\widetilde{N} \nsubseteq Q_{i} \oplus\left(Q_{i}: M\right)$ and for $t<i \leq n$ we have $\widetilde{N} \subseteq Q_{i} \oplus\left(Q_{i}: M\right)$. If $t=0$, then $\widetilde{N}=\cap_{i=1}^{n} \Delta^{Q_{i}}(\widetilde{N})=\cap_{i=1}^{n} \widetilde{M}=\widetilde{M}$ by 2.1 . Consequently, for each $m \in M, m=\Delta(m+0,0+1) \in \Delta(\widetilde{N}, \widetilde{N})=B$, that is, $B=M$ contradicting the properness assumption on $B$.

Thus $t>0$. Note that since $\Delta^{Q_{i}}(\widetilde{N})=\widetilde{M}$ for each $t<i \leq n$, we get $\widetilde{N}=\bigcap_{i=1}^{t} \Delta^{Q_{i}}(\widetilde{N})$ which contains $\bigcap_{i=1}^{t}\left(Q_{i} \oplus\left(Q_{i}: M\right)\right)$ by 2.4. On the other hand, if $t<n$ for any $t<j \leq n$ and by 2.2, 2.4 and 2.1, $\widetilde{N} \subseteq \Delta^{Q_{j}}\left(\Delta^{Q_{j}}(\tilde{N})\right)=\Delta^{Q_{j}}(\widetilde{M})=Q_{j} \oplus\left(Q_{j}: M\right)$. Therefore, $\bigcap_{i=1}^{t}\left(Q_{i} \oplus\left(Q_{i}: M\right)\right) \subseteq Q_{j} \oplus\left(Q_{j}: M\right)$, hence $\bigcap_{i=1}^{t} Q_{i} \subseteq Q_{j}$ which contradicts the minimality of the decomposition of $B$. So $t=n$ and the result is established.

\section{References}

[1] M. Alkan and Y. Tiraş, On prime submodules, Rocky Mount. J. Math. 37 (3), 709$722,2007$.

[2] B. Amini and A. Amini, On strongly superfluous submodules, Comm. Algebra 40 (8), 2906-2919, 2012.

[3] A. Azizi, Radical formula and prime submodules, J. Algebra 307, 454-460, 2007.

[4] A. Azizi, Prime submodules of artinian modules, Taiwanese J. Math. 13 (6B), 20112020, 2009 .

[5] A. Azizi, Radical formula and weakly prime submodules, Glasgow Math. J. 51, 405412, 2009.

[6] A. Azizi and A. Nikseresht, Simplified radical formula in modules, Houston J. Math. 38 (2), 333-344, 2012.

[7] M. Behboodi and H. Koohi, Weakly prime modules, Vietnam J. Math. 32, 185-195, 2004.

[8] S. Çeken and M. Alkan, On Prime submodules and primary decomposition in twogenerated free modules, Taiwanese J. Math. 17 (1), 133-142, 2013.

[9] J. Jenkins and P.F. Smith, On the prime radical of a module over a commutative ring, Comm. Algebra 20 (12), 3593-3602, 1992.

[10] K.H. Leung and S.H. Man, On commutative Noetherian rings which satisfy the radical formula, Glasgow Math. J. 39, 285-293, 1997.

[11] S.H. Man, One dimensional domains which satisfy the radical formula are Dedekind domains, Arch. Math. 66, 276-279, 1996.

[12] S.H. Man, On commutative Noetherian rings which satisfy the generalized radical formula, Comm. Algebra 27 (8), 4075-4088, 1999.

[13] R. McCasland and M.E. Moore, On radicals of submodules of finitely generated modules, Canad. Math. Bull. 29 (1), 37-39, 1986.

[14] R.L. McCasland and P.F. Smith, Zariski spaces of modules over arbitrary rings, Comm. Algebra 34, 3961-3973, 2006.

[15] F. Mirzaei and R. Nekooei, On prime submodules of a finitely generated free module over a commutative ring, Comm. Algebra 44 (9), 3966-3975, 2016.

[16] M.E. Moore and S.J. Smith, Prime and radical submodules of modules over commutative rings, Comm. Algebra 30 (10), 5037-5064, 2002.

[17] A. Nikseresht and A. Azizi, On radical formula in modules, Glasgow Math. J. 53, 657-668, 2011.

[18] A. Nikseresht and A. Azizi, Envelope dimension of modules and the simplified radical formula, Canad. Math. Bull. 56 (4), 683-694, 2013.

[19] A. Parkash, Arithmetical rings satisfy the radical formula, J. Commut. Algebra 4 (2), 293-296, 2012. 
[20] D. Pusat-Yilmaz and P. F. Smith, Modules which satisfy the radical formula, Acta. Math. Hungar. 95, 155-167, 2002.

[21] P. F. Smith, Primary modules over commutative rings, Glasgow Math. J. 43, 103-111, 2001.

[22] Y. Tiraş and M. Alkan, Prime modules and submodules, Comm. Algebra 31 (11), 5263-5261, 2003. 